\title{
Active Hexose Correlated Compound Extends the Lifespan and Increases the Thermotolerance of Nematodes
}

\section{Tetsuya Okuyama, ${ }^{1}$ Emi Yoshigai, ${ }^{2}$ Yukinobu Ikeya,${ }^{3}$ and Mikio Nishizawa ${ }^{2 *}$}

${ }^{1}$ Ritsumeikan Global Innovation Research Organization (R-GIRO), Ritsumeikan University, Kusatsu, Shiga, 525-8577, Japan; ${ }^{2}$ Department of Biomedical Sciences, College of Life Sciences, Ritsumeikan University, Kusatsu, Shiga, 525-8577, Japan; ${ }^{3}$ Department of Pharmacy, College of Pharmaceutical Sciences, Ritsumeikan University, Kusatsu, Shiga, 525-8577, Japan

Corresponding Author: Mikio Nishizawa, MD, PhD, Department of Biomedical Sciences, College of Life Sciences, Ritsumeikan University, Kusatsu, Shiga, 525-8577, Japan

Submission date: April 26, 2013; Acceptance date: June 5, 2013; Publication date: June 7 , 2013

\section{ABSTRACT:}

Background: Active hexose correlated compound (AHCC) is the extract from cultured mycelia of Lentinula edodes, a species of Basidiomycetes mushroom. AHCC contains various polysaccharides, including partially acylated <-1,4-glucan, which is one of its major constituents. The application of AHCC has been markedly increased in complementary and alternative medicine as a functional food because AHCC improved the prognosis of postoperative hepatocellular carcinoma patients. AHCC has anti-inflammatory and antioxidant effects, such as the suppression of nitric oxide production in hepatocytes. AHCC might affect resistance to environmental stress, which is assumed to play a pivotal role in the longevity of many organisms.

Objective: To investigate the effect of AHCC on longevity, we measured the lifespan of the nematode Caenorhabditis elegans, a model animal that is widely used to assess longevity. We also examined the effect of AHCC on resistance to heat stress, i.e., thermotolerance. 
Methods: The lifespan of $C$. elegans animals grown on media in the absence or presence of AHCC at $20^{\circ} \mathrm{C}$ was evaluated. Thermotolerance assays were performed at $35^{\circ} \mathrm{C}$, the restrictive temperature of the animals. The effects of AHCC on lifespan and thermotolerance were analyzed with longevity mutants. Expression levels of stress-related genes, including heat shock genes, were measured by strand-specific reverse transcription-polymerase chain reaction after heat shock.

Results: Wild-type C. elegans animals exhibited a longer mean lifespan by up to $10 \%$ in the presence of AHCC in the growth media than animals in the absence of AHCC. Furthermore, AHCC markedly increased thermotolerance at $35^{\circ} \mathrm{C}$. Epistasis analyses showed that lifespan extension by AHCC at least partly required two longevity-promoting transcription factors: DAF-16 (C. elegans homolog of FOXO) and HSF-1 (C. elegans homolog of heat shock transcription factor 1). After heat shock, AHCC activated the transcription of the heat shock genes, which are the targets of HSF-1. Similarly, the expression of hsf-1 mRNA was elevated following AHCC treatment. Recently, natural antisense transcripts were shown to regulate mRNA stability at the posttranscriptional level. In nematodes, AHCC increased the natural antisense transcript of the $h s f-1$ gene.

Conclusion: AHCC conferred lifespan extension and thermotolerance to C. elegans. Our analyses suggest that the beneficial effects of AHCC on longevity are involved in the activation of at least two transcription factors, DAF-16 and HSF-1, most likely through an antisense transcript-mediated mechanism.

Keywords: longevity, heat stress resistance, HSF, FOXO, heat shock gene

\section{BACKGROUND:}

The functional food active hexose correlated compound (AHCC) is an extract prepared from long-term liquid culture of Lentinula edodes, a species of Basidiomycetes mushroom [1, 2]. AHCC exhibits anti-inflammatory and anti-oxidant effects [3-5] and suppresses the production of the inflammatory mediator nitric oxide (NO) in rat hepatocytes [6]. AHCC reduced not only inducible nitric oxide synthase (iNOS) mRNA but also an iNOS antisense transcript (asRNA) that is involved in iNOS mRNA stabilization [7, 8]. Furthermore, a 10year prospective cohort study indicated that AHCC improved the prognosis of postoperative 
hepatocellular carcinoma patients [2, 9]. Thus, supplementation with AHCC has been increased in complementary and alternative medicine.

Carbohydrates, including polysaccharides and oligosaccharides, are the major components of AHCC, constituting approximately $74 \%$ of the total weight and consisting of polysaccharides, amino acids, lipids, and minerals [1]. The acylated $\langle-1,4$-glucan is assumed to confer the biological activities of AHCC [10]. AHCC inhibits the induction of inducible nitric oxide synthase (iNOS) at the transcriptional level and decreases the iNOS mRNA level by reducing mRNA stability through its $3^{\prime}$ untranslated region [6]. A hydrophilic neutral fraction of AHCC, in which sugars were enriched, inhibited the upregulation of type I interleukin 1 receptor (IL1R1) through the inhibition of Akt phosphorylation [11].

Aging is hormonally regulated by various pathways that have been conserved during evolution, and the best understood pathway influencing lifespan is the insulin/insulin-like growth factor 1 (IGF-1) signaling pathway [12]. Mutations in the daf-2 gene, which encodes an insulin/IGF-1 receptor ortholog, increase the lifespan of the nematode Caenorhabditis elegans. Furthermore, studies in mice lacking the insulin receptor or IGF-1 receptor suggest that the ability of insulin/IGF-1 signaling to influence lifespan has been distributed to both insulin and IGF-1 receptors during evolution. The lifespan extension caused by daf-2 mutations in $C$. elegans requires the activity of daf-16, which encodes the forkhead transcription factor O (FOXO) [12]. This transcription factor plays key roles in insulin/IGF-1 signaling to modulate aging in many organisms.

Heat shock transcription factor 1 (HSF-1) delays aging and extends the lifespan of $C$. elegans [12]. In daf-2 mutants, HSF-1 promotes longevity by activating heat shock genes that encode small heat shock proteins, such as hsp-12.6 and hsp-16.49 [13], as well as the hsp70 family protein F44E5.4 [13]. Similar to DAF-16, HSF-1 is essential for daf-2 mutations to extend lifespan. Another transcription factor, SKN-1, is also involved in the insulin/IGF-1 signaling pathway and extracellular signal-regulated kinase (ERK)-mitogen-activated protein kinase (MAPK) pathway, both of which regulate longevity [14].

C. elegans have been widely used as a model to assess longevity because this invertebrate animal has a much shorter lifespan (i.e., a few weeks) than mammalian species. In addition, tolerance against heat stress-i.e., thermotolerance-highly correlates with the lifespan of $C$. elegans because closely related molecular mechanisms are assumed to be involved in this species' ability to defend itself from heat stress and the damage caused by aging [15]. It is plausible that foods are the factors that influence longevity and thermotolerance. The functional food AHCC might affect resistance to environmental stress, which plays a pivotal role in the longevity of many organisms. To investigate the effect of 
AHCC on longevity, we investigated the lifespan of $C$. elegans and examined the effect of AHCC on resistance to thermotolerance.

\section{MATERIALS AND METHODS:}

Materials: AHCC freeze-dried powder was generously provided by Amino Up Chemical Co., Ltd. (Sapporo, Hokkaido, Japan). Fermented liquid media of Lentinula edodes was processed by separation, concentration, sterilization, and freeze-drying to the end compound of AHCC under the QMS standards of ISO 9001:2008, and ISO 22000:2005, and Japan Health and Nutrition Food Association GMP Program for Dietary Supplements.

Measurement of $\alpha$-glucan content: The $\alpha$-glucan content in each lot of AHCC powder was measured as glucose liberated by enzymatic digestion. AHCC powder ( $5 \mathrm{mg}$ ) was dissolved in $1 \mathrm{ml}$ of $100 \mathrm{mM}$ sodium acetate $\left(\mathrm{pH} \mathrm{4.8)}\right.$ and preincubated at $50^{\circ} \mathrm{C}$ for $5 \mathrm{~min}$. Next, $1 \mathrm{ml}$ of amyloglucosidase (EC 3.2.1.3) from Aspergillus niger (70 units/ml; Sigma-Aldrich: Fluka, St. Louis, MI, USA) was added, and the reaction mixture was further incubated at $50^{\circ} \mathrm{C}$ for $60 \mathrm{~min}$. An aliquot $(10 \mu \mathrm{l})$ of the reaction mixture was subjected to glucose measurement using Glucose C-II Test Wako Kits (Wako Pure Chemical Industries, Ltd., Osaka, Japan). Glucose at known concentrations was used as a standard to calculate the $\alpha$-glucan content.

Measurement of NO production: AHCC powder was added with $1 \mathrm{nM}$ IL-1 $\beta$ to the medium for primary cultured rat hepatocytes. After $8 \mathrm{~h}$, the NO level (as nitrite) in the medium was measured using the Griess method as previously described [6].

Strains of C. elegans: All strains were maintained at $20^{\circ} \mathrm{C}$ on nematode growth medium and fed on kanamycin-killed Escherichia coli strain OP50 as previously described [16]. Bacteria were spread on top of the agar plates containing $50 \mu \mathrm{g} / \mathrm{ml}$ kanamycin, and the animals were transferred onto the bacterial lawn. The strains used in this study were as follows: Bristol N2, a wild-type strain; GR1307daf-16(mgDf50), a daf-16(-) mutant, in which the daf-16 gene has been disrupted; and PS3551hsf-1(sy441), an hsf-1(-) mutant, in which $h s f-1$ has been disrupted $[17,18]$.

Egg count assay: The wild-type animals were synchronized and then grown on agar plates containing $2 \mathrm{mg} / \mathrm{ml}$ AHCC or on control plates in the absence of AHCC. The non-gravid animals were individually placed on separate agar plates in the presence or absence of AHCC 
(Time 0). Animals were transferred to new plates, and the eggs laid on each plate were counted as the resulting brood size.

Lifespan assay: The synchronized young adult animals grown on the plates containing AHCC were transferred to plates containing $25 \mu \mathrm{M}$ 5-fluoro-2'-deoxyuridine (Wako Pure Chemical Industries, Ltd.), which inhibits DNA replication to prevent the growth of progeny (Day 0). Death was scored as the absence of any movement and failure to move at all after light punches as previously described [14].

Thermotolerance assay: The synchronized young adults were transferred from $20^{\circ} \mathrm{C}$ to $35^{\circ} \mathrm{C}$, the restrictive temperature of the nematodes. Death of each animal was assessed after the temperature shift.

Reverse transcription-polymerase chain reaction (RT-PCR): Total RNA was isolated from the wild-type young adults and purified using TURBO DNA-free kits (Applied Biosystems, Austin, TX, USA). The cDNA was strand-specifically reverse transcribed at $47^{\circ} \mathrm{C}$ using an oligo(dT) primer for mRNA as described previously [19]. To avoid the amplification of self-primed transcripts [20], the cDNA was reverse transcribed at $53^{\circ} \mathrm{C}$ using an $h s f-1$-specific sense primer for the hsf-1 asRNA. The primers used are shown in Table 1. Relative mRNA levels were measured by real-time PCR, and values were normalized by the levels of the internal control eft-4 (translation elongation factor $1 \alpha$ homolog) mRNA.

Table 1. Primers used in this study

\begin{tabular}{|c|c|c|c|c|}
\hline $\begin{array}{l}\text { Transcript } \\
\text { to be detected }\end{array}$ & Sequence $\left(5^{\prime} \longrightarrow 3^{\prime}\right)$ & $\begin{array}{l}\mathrm{RT} / \\
\mathrm{PCR}^{1}\end{array}$ & Direction & $\begin{array}{l}\text { cDNA } \\
(\mathrm{bp})^{2}\end{array}$ \\
\hline \multirow[t]{2}{*}{ eft-4 mRNA } & GTGACCACTGAAGTCAAGTCCGT & PCR & Forward & 293 \\
\hline & CGACGATCGACCTTCTCCTTAAG & PCR & Reverse & \\
\hline \multirow[t]{2}{*}{ F44E5.4 mRNA } & TCAGAATGGAAAGGTTGAGATCC & PCR & Forward & 292 \\
\hline & CTCTTCTGGATTGAATTGCCTC & PCR & Reverse & \\
\hline \multirow[t]{2}{*}{ hsf-1 mRNA } & GTTGAATATGTACGGCTTCCGAA & PCR & Forward & 293 \\
\hline & TATCGCGATTTTCTTTTGTAAGCTT & PCR & Reverse & \\
\hline
\end{tabular}




\begin{tabular}{lllll} 
hsf-1 asRNA & ATTGATTTTTTTTTTGAACG & RT & Forward & \\
& GCTCAAAATTCCTCTCTTTTCC & PCR & Forward & 253 \\
& TATTCCGAAAAGTTTCGGGGGT & PCR & Reverse & \\
hsp-12.6 mRNA & GAGTTGTCAATGTCCTCGACGA & PCR & Forward & \multirow{2}{*}{188} \\
& CTTTGGAAGTTTGTAACAACGAG & PCR & Reverse & \\
& & & & \\
hsp-16.49 mRNA & CAATATTGGAGAGATTGTAAATGAC & PCR & Forward & 317 \\
& GAGTTAGTCTTCTTTGGAGCCTC & PCR & Reverse &
\end{tabular}

\footnotetext{
${ }^{1}$ An oligo(dT) primer used for reverse transcription (RT) to synthesize complementary DNA (cDNA) to mRNA. A sense primer used for RT to synthesize cDNA to the hsf-1 antisense transcript (asRNA). Primer pairs used for PCR. ${ }^{2}$ The size of the cDNA fragment amplified by each pair of PCR primers is shown in base pairs (bp). eft-4, translation elongation factor 4; hsf-1, heat shock transcription factor 1; and hsp, heat shock protein.
}

Statistical analysis: The results in the figures are representative of at least three independent experiments that yielded similar results. The values are presented as the means \pm standard deviation (SD). Differences were analyzed using Student's $t$-test. Statistical significance was set at $P<0.05$ and $P<0.01$.

\section{RESULTS:}

\section{Qualification of the AHCC lots}

AHCC consists of carbohydrates and several ingredients [1] (Table 2), and oligosaccharides are assumed to confer the biological activities of AHCC $[10,11]$. Because $\alpha$-glucan is a major constituent of carbohydrates in AHCC, the $\alpha$-glucan content may be a standard to qualify AHCC lots. Therefore, we measured the $\alpha$-glucan content by the method with amyloglucosidase as described in the Materials and Methods section. This enzymatic analysis indicated that the $\alpha$-glucan content was $28.9 \pm 2.4 \%$ (weight) of the AHCC freezedried powder $(n=35)$, suggesting that deviation in the content among the AHCC lots was small. By contrast, the $\beta$-glucan content was very low [1]. 
Table 2. Ingredients of AHCC freeze-dried powder.

\begin{tabular}{|c|c|c|}
\hline Ingredient & Content $(\%)$ & Analysis method \\
\hline Protein $^{1}$ & 13.1 & Kjeldahl method \\
\hline Fats $^{1}$ & 2.2 & Acid decomposition method \\
\hline Dietary fiber $^{1}$ & 2.1 & Enzymatic-gravimetric method \\
\hline Ash contents ${ }^{1}$ & 8.9 & Direct ashing method \\
\hline Carbohydrate $^{1}$ & 71.2 & As per the balance \\
\hline$\alpha$-Glucan ${ }^{2}$ & $28.9 \pm 2.4$ & Enzymatic method (this study) \\
\hline$\beta$-Glucan ${ }^{1}$ & 0.2 & Enzymatic method, ELISA \\
\hline
\end{tabular}

${ }^{1}$ Miura et al. [1]. ${ }^{2}$ Represented as the mean \pm standard deviation $(n=35)$.

Next, we measured the biological activity of AHCC. Because the AHCC freeze-dried powder suppresses NO production in IL-1 $\beta$-treated hepatocytes [6], we measured the suppression activities of NO production by AHCC. All the lots showed comparable suppression activities (data not shown), indicating an average $50 \%$ inhibitory concentration $\left(\mathrm{IC}_{50}\right)$ of $3.75 \pm 0.15 \mathrm{mg} / \mathrm{ml}(n=8)$.

This data confirmed that the deviation of quality among the lots of AHCC powder was small. Therefore, we chose one among these examined lots and used it for subsequent experiments with $C$. elegans.

\section{AHCC does not affect the brood size of C. elegans}

To investigate whether AHCC attenuated the brood of C. elegans, we added AHCC to the growth media and estimated the brood size of the wild-type N2 strain. AHCC treatment (2 $\mathrm{mg} / \mathrm{ml})$ did not affect the total brood size per animal $(P=0.078)$ when comparing the total brood size of the control animals in the absence of AHCC (Fig. 1A). In addition, counting of progeny revealed that a similar number of progeny was produced by the negative controls (i.e., animals in the absence of AHCC (Fig. 1B)). These results suggest that AHCC had a small effect on the reproduction of $C$. elegans, although AHCC treatment slightly altered the reproductive schedule. 
A

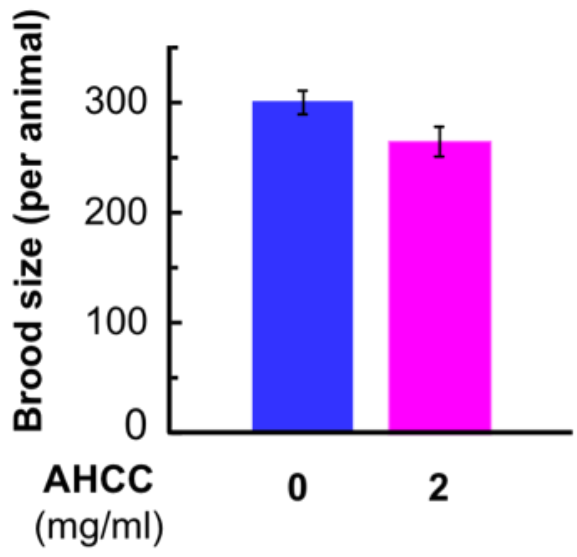

B

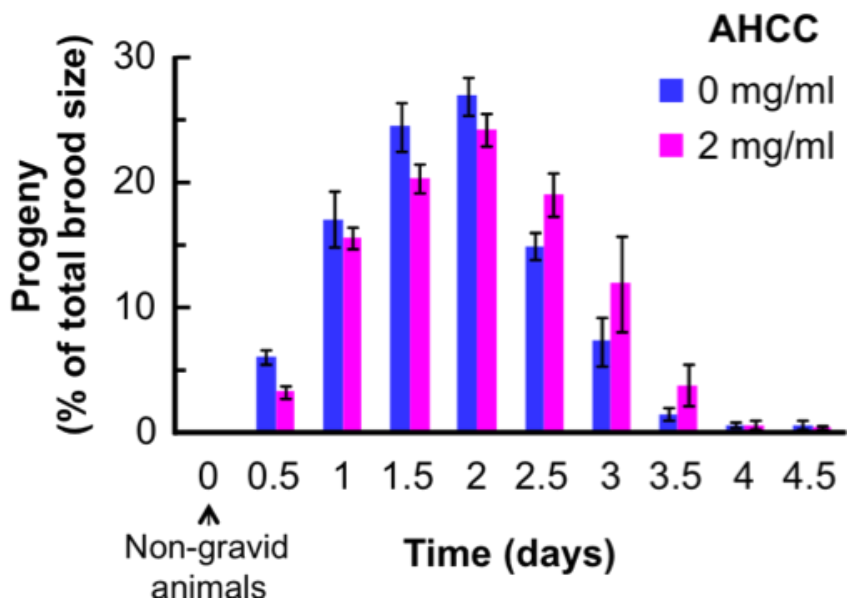

Fig. 1. AHCC treatment does not affect the brood size of C. elegans. (A) The brood sizes of the wild-type N2 strain. An egg count assay was performed. Total brood sizes per animal in the absence or presence of AHCC are shown as the means \pm standard deviation (SD). (B) Progeny of the wildtype strain. Progeny of non-gravid animals are shown as the means \pm SD as a percentage of the total brood size.

\section{AHCC extends the lifespan of wild-type C. elegans}

Next, we added AHCC to the growth medium at different concentrations and estimated the lifespan of the wild-type nematodes. The fraction alive was measured, and survival curves were drawn (Fig. 2A). The wild-type strain placed on the agar plates containing 1, 2, or 4 $\mathrm{mg} / \mathrm{ml} \mathrm{AHCC}$ lived longer than the negative controls in the absence of AHCC $(0 \mathrm{mg} / \mathrm{ml})$. We calculated the mean lifespans and compared them with the negative controls. As shown in Fig. 2B, wild-type animals exhibited a significantly longer mean lifespan in the presence of AHCC.

A

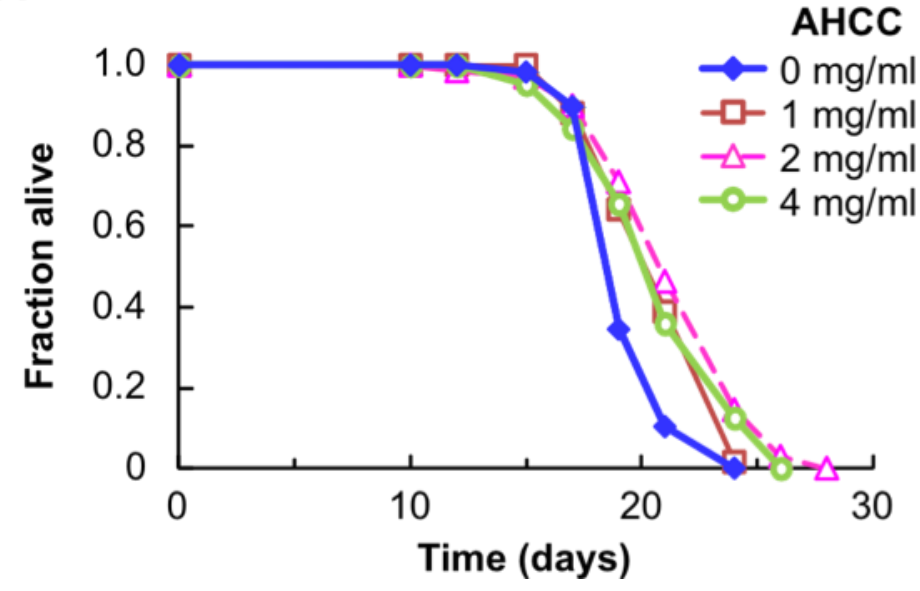

B

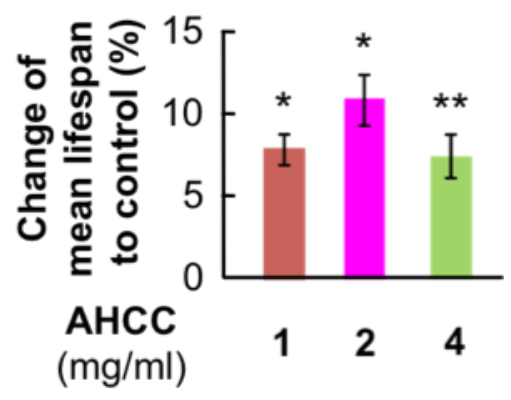


Fig. 2. Wild-type nematodes live longer in the presence of AHCC. (A) Survival curves of the wild-type strain in the presence of AHCC. The fraction alive is shown. (B) Changes of the mean lifespan relative to the negative control animals $(0 \mathrm{mg} / \mathrm{ml}$ AHCC $)$. Changes of the mean lifespan relative to the controls are shown as the means $\pm \mathrm{SD} .{ }^{*} P<0.05, * * P<0.01$ versus the negative controls.

\section{DAF-16 is required for AHCC to extend lifespan}

Next, we examined the effects of the longevity-promoting transcription factors DAF-16 and HSF-1 in the insulin/IGF-1 signaling pathway. Two animal mutants, in which the daf-16 or $h s f-1$ gene was disrupted, were used for the lifespan assay. When the daf-16(-) mutant was used, AHCC did not increase its lifespan (Fig. 3A), suggesting that DAF-16 is necessary for the extension of lifespan by AHCC. By contrast, AHCC caused a slight but significant extension of the lifespan in the hsf-1(-) mutant (Fig. 3B and 3C). These results indicate that the lifespan extension by AHCC at least required DAF-16.

A

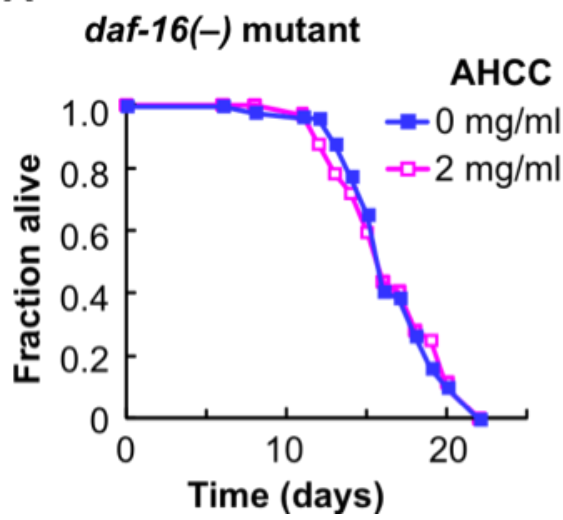

B

\section{hsf-1(-) mutant}

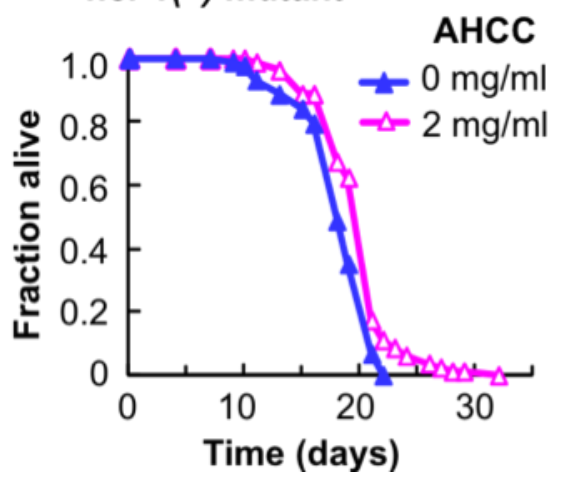

C

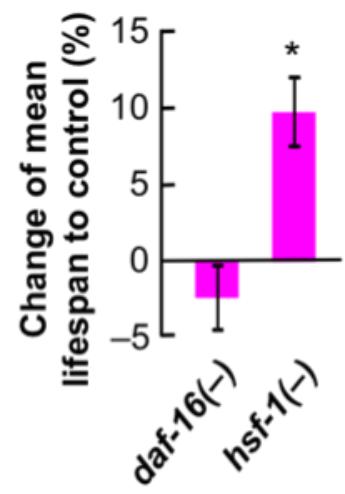

Fig. 3. AHCC does not affect the lifespan of the daf-16(-) mutant. (A) Survival curves of the daf-16(-) mutant in the absence and presence of AHCC. The fraction alive is shown. (B) Survival curves of the $h s f-1(-)$ mutant in the absence and presence of the AHCC fraction alive is shown. (C) Changes of the mean lifespan versus the negative controls $(0 \mathrm{mg} / \mathrm{ml}$ AHCC $)$ are shown as the means \pm SD. $* P<0.05$ versus the negative controls.

\section{AHCC enhances the thermotolerance of C. elegans through HSF-1}

The effects of AHCC on the thermotolerance of $C$. elegans were examined. Because the lethal temperature of the wild-type $\mathrm{N} 2$ strain is $35^{\circ} \mathrm{C}$, we transferred the animals from $20^{\circ} \mathrm{C}$ to $35^{\circ} \mathrm{C}$, and the fraction alive was measured in the absence and presence of AHCC. As shown in Fig. 4A, AHCC prominently increased the thermotolerance of the wild-type 
animals at $35^{\circ} \mathrm{C}$. When the average time of death after the temperature shift to $35^{\circ} \mathrm{C}$ was calculated, the viability of AHCC-treated animals was significantly higher than that of the negative controls $(0 \mathrm{mg} / \mathrm{ml}$ AHCC). The mean time of death in the presence of AHCC was $24 \%$ longer than that in the absence of AHCC (negative controls).

A

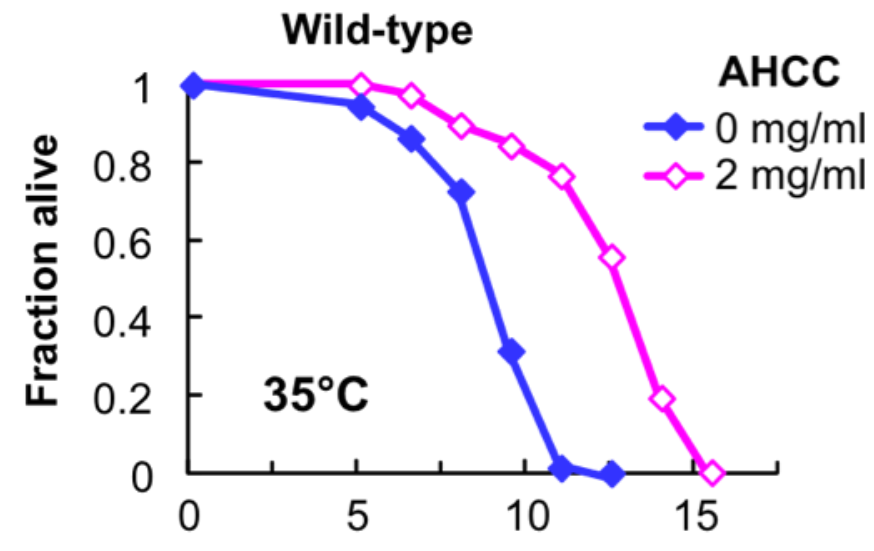

Time after temperature shift (h)

B

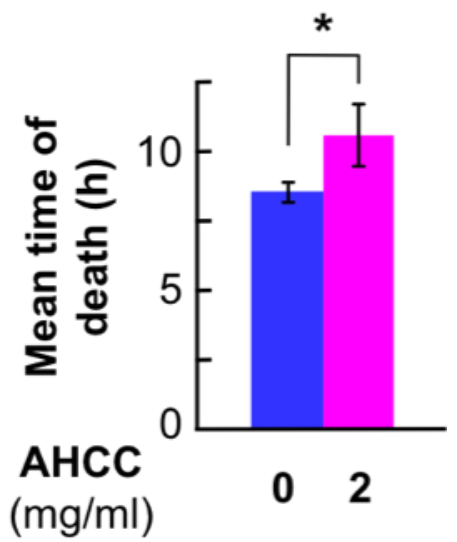

Fig. 4. AHCC increases the thermotolerance of the wild-type animals. (A) Survival curves of the wild-type strain in the absence or presence of AHCC. The fraction alive after the temperature shift to $35^{\circ} \mathrm{C}$ is shown. (B) The average time to death after the temperature shift. Values are means \pm SD. $* P<0.05$ versus the negative controls.

To investigate the effects of the longevity-related transcription factors DAF-16 and HSF-1 on thermotolerance, the nematode mutants were used. When the $h s f-1(-)$ mutant was used, AHCC did not increase its viability (Fig. 5A), suggesting that HSF-1 is necessary for AHCC-enhanced thermotolerance. By contrast, AHCC increased viability in the daf-16(-) mutant, similar to that in the wild-type strain (Fig. 5B), suggesting that DAF-16 is not essential for AHCC-enhanced thermotolerance. These data indicate that AHCC-enhanced thermotolerance at least required HSF-1.

A

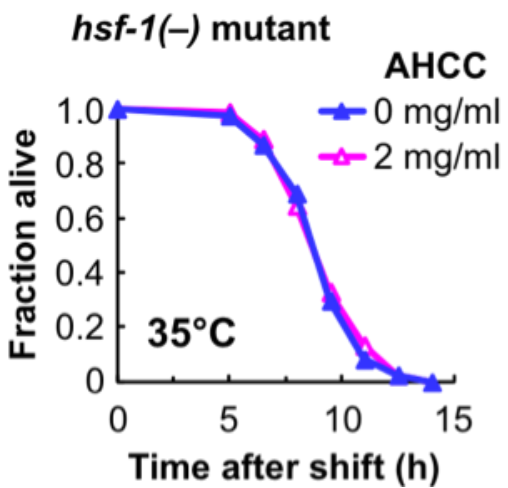

B

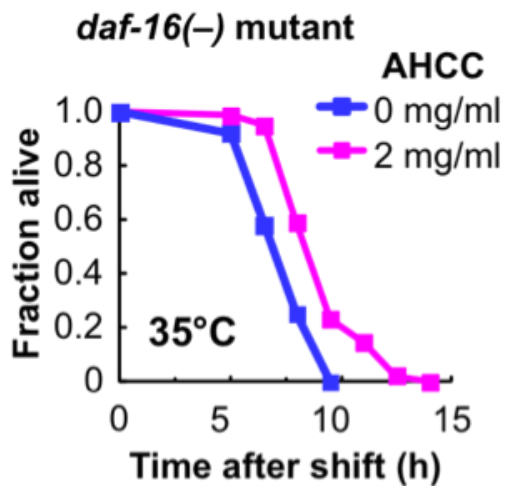

C

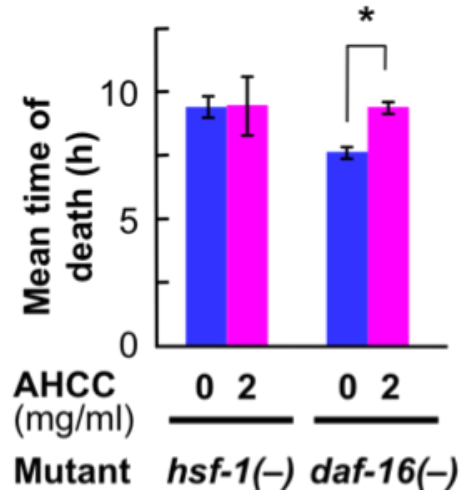


Fig. 5. AHCC did not increase the thermotolerance of the $h s f-1(-)$ mutant. (A) Survival curves of the $h s f-1(-)$ mutant in the absence and presence of AHCC. The fraction alive after the transfer to the lethal temperature $\left(35^{\circ} \mathrm{C}\right)$ is shown. (B) Survival curves of the $d a f-16(-)$ mutant in the absence and presence of AHCC. The fraction alive after the temperature shift is shown. (C) The mean time to death after the shift to $35^{\circ} \mathrm{C}$. Values represent means $\pm \mathrm{SD}$. $* P<0.05$ versus the negative controls.

\section{AHCC increases the levels of both hsf-1 mRNA and asRNA}

To examine the effects of AHCC on $h s f-1$ gene expression, hsf-1 mRNA was detected by RT and real-time PCR. The addition of AHCC elevated the hsf-1 mRNA level in the wild-type animals (Fig. 6A).

Recently, it was reported that the asRNA transcribed from a gene (i.e., the natural antisense transcript) regulates mRNA stability at the posttranscriptional level [7, 21-23]. Therefore, we examined whether an asRNA was transcribed from the $h s f-l$ gene. Primer sets to detect asRNA that corresponded to the $3^{\prime}$ untranslated region of hsf-1 mRNA were designed (Table 1), and strand-specific RT-PCR was performed. As shown in Fig. 6B, hsf-1 asRNA was detected in the absence of AHCC, and the level of hsf-1 asRNA increased in the presence of AHCC.

A

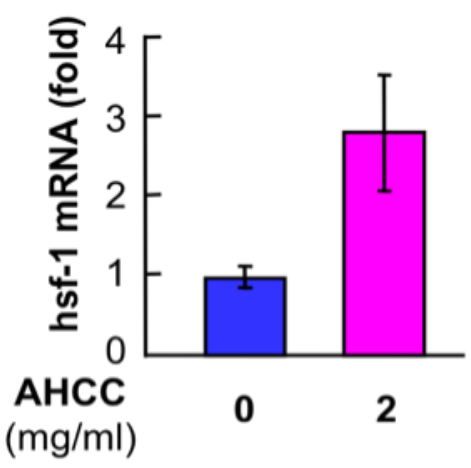

B

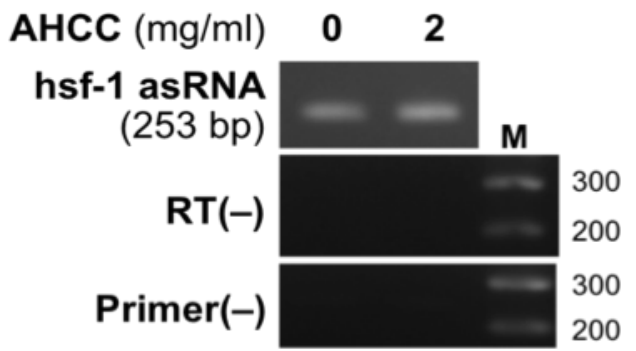

C

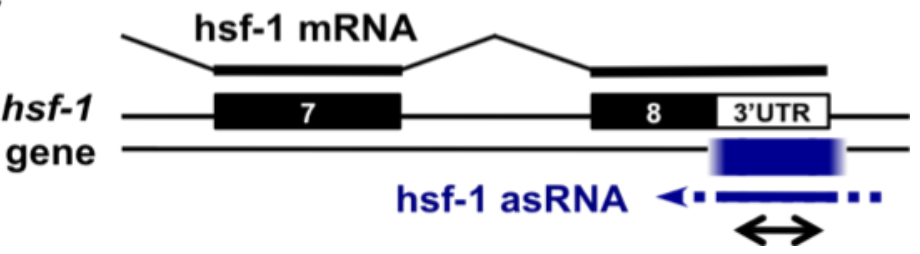

Fig. 6. AHCC increases the levels of both hsf-1 mRNA and asRNA. (A) The levels of hsf-1 mRNA in the wild-type strain in the absence and presence of AHCC. The hsf-1 mRNA levels (means $\pm \mathrm{SD}$ ) were measured by real-time PCR and normalized to the levels of eft- 4 mRNA (internal control). ${ }^{*} P<0.05$ versus the negative controls. (B) Expression of hsf-1 asRNA. Strand-specific RTPCR was performed to detect hsf-1 asRNA. RT(-), a PCR control without RT to monitor the genomic DNA contamination. Primer(-), a control without an hsf-1-specific sense primer during RT to monitor self-primed cDNA. M, DNA size markers in base pairs. (C) The structure and expression of the $h s f-1$ gene. Exon 8 for hsf-1 mRNA and the transcribed regions for hsf-1 asRNA are depicted. Boxes 
indicate the transcribed regions of the gene. The region amplified by PCR is indicated by a doubleheaded arrow beneath the 3' untranslated region (3'UTR), and hsf-1 asRNA is indicated by an arrow.

\section{AHCC enhances the expression of heat shock genes}

It was expected that AHCC, after heat shock, would enhance the expression of heat shock genes, whose promoters HSF-1 binds. Therefore, we examined the expression of these genes (hsp-12.6, hsp-16.49, and F44E5.4) in the wild-type animals that were heat shocked by the temperature shift to $35^{\circ} \mathrm{C}$. The levels of hsp-12.6, hsp-16.49, and F44E5.4 mRNAs increased by the heat shock in a time-dependent manner (Fig. 7). After the temperature shift, AHCC significantly enhanced the levels of hsp-12.6, hsp-16.49, and F44E5.4 mRNAs.

A

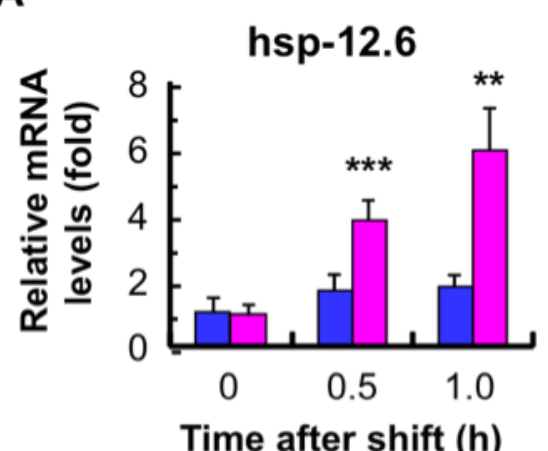

B

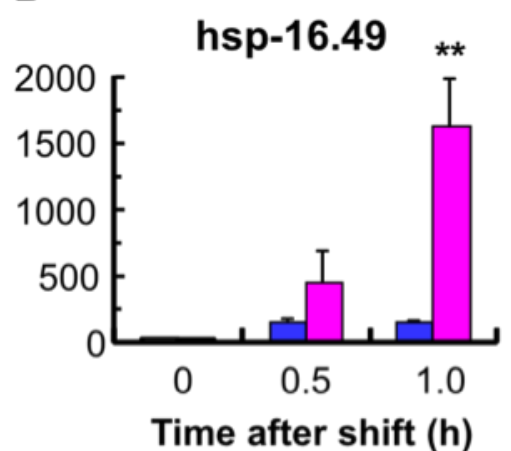

C

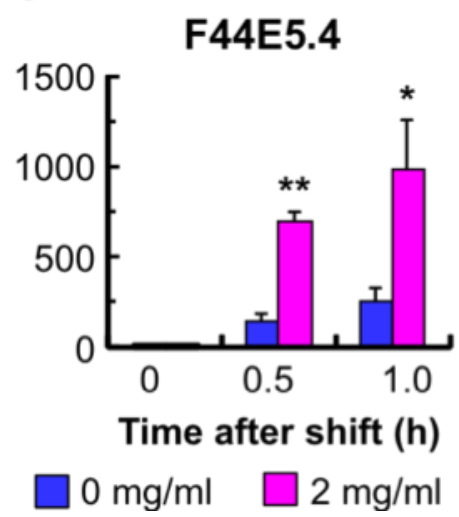

Fig. 7. AHCC increases the expression of heat shock genes. Heat shock gene expression in the heat shocked wild-type nematodes was analyzed. After the temperature shift to $35^{\circ} \mathrm{C}$ of the wild-type strain in the absence and presence of AHCC, the levels of hsp-12.6 (A), hsp-16.49 (B), and F44E5.4 (C) mRNAs were analyzed by real-time RT-PCR. The values were normalized to those of eft-4 mRNA (internal control). The normalized value at $0 \mathrm{~h}$ after heat shock in the absence of AHCC was set at 1 , and relative mRNA levels (means $\pm \mathrm{SD}$ ) are shown in fold values. ${ }^{*} P<0.05$, $* * P<0.01$, *** $P<0.001$ versus control animals heat shocked for the same time

\section{DISCUSSION:}

The present study demonstrates that AHCC extended the lifespan of C. elegans and increased its thermotolerance. Various functional and medical foods from fruit and plant extracts (e.g., blueberry and Ginkgo biloba extract), as well as their constituents (i.e., catechins and caffeic acid), have been reported to promote lifespan in invertebrate models [24]. Our study is the first to demonstrate that AHCC, derived from the mushroom Lentinula edodes, contributes to lifespan extension in C. elegans. 
The insulin/IGF-1 signaling pathway is a major mechanism involved in regulating the lifespan of C. elegans [12], and our study suggests that this pathway is highly involved in the lifespan extension via AHCC. The lifespan extension caused by daf-2 mutations in C. elegans requires the activity of daf-16, a FOXO transcription factor [12]. The analyses with the daf16 or $h s f-1$ mutants of the nematodes clearly demonstrated that the lifespan extension by AHCC at least partly requires DAF-16 (Fig. 3).

Thermotolerance is highly correlated with C. elegans lifespan, and the molecular mechanism of thermotolerance seems to be closely related to that of aging [15]. AHCC prominently increased the thermotolerance of the wild-type animals at the lethal temperature $35^{\circ} \mathrm{C}$, and the viability of AHCC-treated animals was significantly higher than that of the negative controls (Fig. 4). Thermotolerance assays with the daf-16(-) mutant indicated that the AHCC-enhanced thermotolerance required another longevity-promoting transcription factor, namely, HSF-1. The addition of AHCC elevated the levels of hsf-1 mRNA and hsf-1 asRNA (Fig. 6). Therefore, it is possible that hsf-1 asRNA posttranscriptionally regulates the stability of hsf-1 mRNA, similar to that of iNOS, cytokine, and chemokine mRNAs [7, 22, 23]. AHCC markedly increased the expression of hsp-12.6, hsp-16.49, and F44E5.4 mRNAs (Fig. 7), and HSF-1 binds to the promoters of these heat shock genes [13]. These results suggest that AHCC enhances thermotolerance primarily through HSF-1.

Actions of AHCC on longevity and stress resistance as thermotolerance are summarized as a putative model (Fig. 8). The positive effects of AHCC on lifespan extension and thermotolerance are involved in the activation of at least two transcription factorsDAF-16 and HSF-1 - both of which are included in the insulin/IGF-1 pathway. However, the molecular mechanism underlying thermotolerance seems to be closely related to that of aging, but it is not identical.

A receptor for the acylated $\alpha-1,4$-glucan in AHCC is not identified, although this $\alpha$ glucan is assumed to confer the biological activities of AHCC [10, 11]. Immunological properties of $\alpha$-glucans have been much less studied than those of $\beta$-glucans. There are only two reports about $\alpha$-glucan receptors: An $\alpha$-glucan from the cell wall of Pseudallescheria boydii, a saprophytic fungus, binds to toll-like receptor 2 (TLR2) [25]; and an $\alpha$-glucan from Mycobacterium tubereculosis cell wall binds to a C-type lectin, which is identified dendritic cell-specific intercellular adhesion molecule-3-grabbing non-integrin (DC-SIGN) [26]. Similar to these $\alpha$-glucans, the acylated $\alpha-1,4$-glucan in AHCC may bind to its putative receptor and affect the functions of DAF-16 (FOXO) and HSF-1 in the insulin/IGF-1 signaling pathway in the nematode and mammals. It remains to be studied how the acylated $\alpha-1,4$-glucan affect the insulin/IGF-1 signaling pathway. 
In rat hepatocytes, AHCC has an anti-inflammatory effect to suppress NO production and iNOS expression [6,11], and IGF-1 inhibits the induction of iNOS in a murine liver failure model [27]. By contrast, C. elegans lacks its own nitric oxide synthase, and NO produced by bacteria extends the C. elegans lifespan [28]. Therefore, it is clear that results using an invertebrate model in this study cannot directly apply to mammals. Further studies are required to elucidate the action of AHCC and other nutraceuticals as well as the relationship between the lifespan extension in C. elegans and healthy aging in mammals.

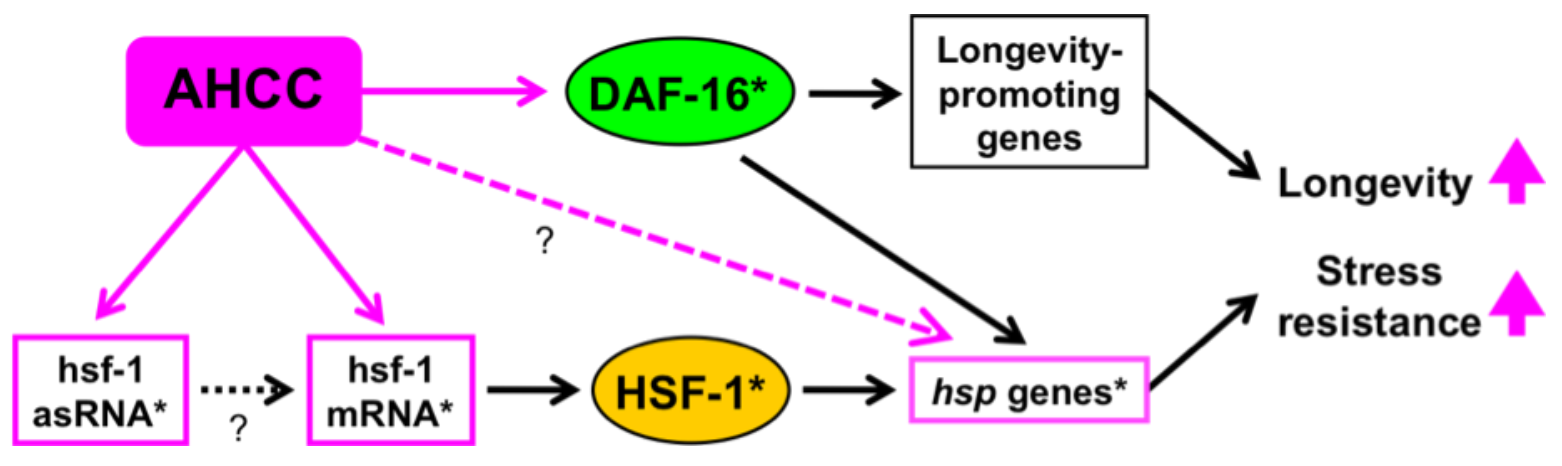

Fig. 8. A putative model of the action of AHCC in C. elegans. Factors affected by AHCC are depicted. Longevity-promoting genes regulated by DAF-16 include the metallothionein $(m t l-1)$ and superoxide dismutase (sod-3) genes [13]. *, factors examined in this study.

\section{CONCLUSION:}

AHCC conferred lifespan extension and thermotolerance to C. elegans. Our study suggests that the positive effects of AHCC on longevity and thermotolerance are involved in the insulin/IGF-1 signaling pathway through two transcription factors, DAF-16 and HSF-1, most likely through an asRNA-mediated mechanism.

Competing Interests: This work was supported in part by research grants from Amino Up Chemical Co., Ltd., the R-GIRO of Ritsumeikan University and Japan Society for the Promotion of Science (JSPS) KAKENHI Grant Number 24657121.

Authors' contributions: All authors contributed to this study.

Abbreviations: AHCC, active hexose correlated compound; IGF-1, insulin-like growth factor 1; FOXO, forkhead transcription factor O; HSF-1, heat shock factor 1; hsp, heat shock protein; RT, reverse transcription; PCR, polymerase chain reaction. 
Acknowledgements and Funding: The authors wish to thank Drs. Tadayoshi Okumura, Yoshito Tanaka, and Masaharu Oishi (Kansai Medical University, Hirakata, Japan) for measuring the NO production; Drs. Tomohiro Ito, Jun Nakahigashi, and Koji Wakame (Amino Up Chemical Co., Ltd.) for providing us with a protocol for measuring the $\alpha$-glucan content and partly measuring it; and Mrs. Noriko Kanazawa for her secretarial assistance. This work was supported in part by research grants from Amino Up Chemical Co., Ltd., the R-GIRO of Ritsumeikan University and Japan Society for the Promotion of Science (JSPS) KAKENHI Grant Number 24657121.

\section{REFERENCES:}

1. Miura T, Kitadate K, Nishioka H, Wakame K. Basic and clinical studies on Active Hexose Correlated Compound. in: Biotechnology in Functional Foods and Nutraceuticals. Bagchi D, Lau FC, Ghosh DK (eds.). CRC Press Taylor and Francis Group. 2010; 51-59.

2. Shah SK, Walker PA, Moore-Olufemi SD, Sundaresan A, Kulkarni AD, Andrassy RJ. An evidence-based review of a Lentinula edodes mushroom extract as complementary therapy in the surgical oncology patient. JPEN J Parenter Enteral Nutr 2011; 35: 449458.

3. Ritz BW, Nogusa S, Ackerman EA, Gardner EM. Supplementation with active hexose correlated compound increases the innate immune response of young mice to primary influenza infection. J Nutr 2006; 136: 2868-2873.

4. Daddaoua A, Martínez-Plata E, López-Posadas R, Vieites JM, González M, Requena P, Zarzuelo A, Suárez MD, de Medina FS, Martínez-Augustin O. Active hexose correlated compound acts as a prebiotic and is antiinflammatory in rats with hapten-induced colitis. J Nutr 2007; 137: 1222-1228.

5. Terakawa N, Matsui Y, Satoi S, Yanagimoto H, Takahashi K, Yamamoto T, Yamao J, Takai S, Kwon AH, Kamiyama Y. Immunological effect of active hexose correlated compound (AHCC) in healthy volunteers: a double-blind, placebo-controlled trial. Nutr Cancer 2008; 60: 643-651.

6. Matsui K, Kawaguchi Y, Ozaki T, Tokuhara K, Tanaka H, Kaibori M, Matsui Y, Kamiyama Y, Wakame K, Miura T, Nishizawa M, Okumura T. Effect of active hexose correlated compound on the production of nitric oxide in hepatocytes. JPEN J Parenter Enteral Nutr 2007; 31: 373-380. 
7. Matsui K, Nishizawa M, Ozaki T, Kimura T, Hashimoto I, Yamada M, Kaibori M, Kamiyama Y, Ito S, Okumura T. Natural antisense transcript stabilizes inducible nitric oxide synthase messenger RNA in rat hepatocytes. Hepatology 2008; 47: 686-697.

8. Nishizawa M, Okumura T, Ikeya Y, Kimura T. Regulation of inducible gene expression by natural antisense transcripts. Front Biosci 2012; 17: 938-958.

9. Matsui Y, Uhara J, Satoi S, Kaibori M, Yamada H, Kitade H, Imamura A, Takai S, Kawaguchi Y, Kwon AH, Kamiyama Y. Improved prognosis of postoperative hepatocellular carcinoma patients when treated with functional foods: a prospective cohort study. J Hepatol 2002; 37: 78-86.

10. Ye SF, Ichimura K, Wakame K, Ohe M. Suppressive effects of Active Hexose Correlated Compound on the increased activity of hepatic and renal ornithine decarboxylase induced by oxidative stress. Life Sci 2003; 74: 593-602.

11. Matsui K, Ozaki T, Oishi M, Tanaka Y, Kaibori M, Nishizawa M, Okumura T, Kwon AH. Active hexose correlated compound inhibits the expression of proinflammatory biomarker iNOS in hepatocytes. Eur Surg Res 2011; 47: 274-283.

12. Kenyon C. The plasticity of aging: insights from long-lived mutants. Cell 2005; 120: $449-460$.

13. Hsu AL, Murphy CT, Kenyon C. Regulation of aging and age-related disease by DAF16 and heat-shock factor. Science 2003; 300: 1142-1145.

14. Okuyama T, Inoue H, Ookuma S, Satoh T, Kano K, Honjoh S, Hisamoto N, Matsumoto K, Nishida E. The ERK-MAPK pathway regulates longevity through SKN-1 and insulinlike signaling in Caenorhabditis elegans. J Biol Chem 2010; 285: 30274-30281.

15. Muñoz MJ. Longevity and heat stress regulation in Caenorhabditis elegans. Mech Ageing Dev 2003; 124: 43-48.

16. Brenner S. The genetics of Caenorhabditis elegans. Genetics 1974; 77: 71-94.

17. Ogg S, Paradis S, Gottlieb S, Patterson GI, Lee L, Tissenbaum HA, Ruvkun G. The Fork head transcription factor DAF-16 transduces insulin-like metabolic and longevity signals in C. elegans. Nature 1997; 389: 994-999.

18. Hajdu-Cronin YM, Chen WJ, Sternberg PW. The L-type cyclin CYL-1 and the heatshock-factor HSF-1 are required for heat-shock-induced protein expression in Caenorhabditis elegans. Genetics 2004; 168: 1937-1949.

19. Nishizawa M, Nakajima T, Yasuda K, Kanzaki H, Sasaguri Y, Watanabe K, Ito S. Close kinship of human 20alpha-hydroxysteroid dehydrogenase gene with three aldo-keto reductase genes. Genes Cells 2000; 5: 111-125. 
20. Haddad F, Qin AX, Giger JM, Guo H, Baldwin KM. Potential pitfalls in the accuracy of analysis of natural sense-antisense RNA pairs by reverse transcription-PCR. BMC Biotechnol 2007; 7: 21.

21. Faghihi MA, Wahlestedt C. Regulatory roles of natural antisense transcripts. Nat Rev Mol Cell Biol 2009; 10: 637-643.

22. Yoshigai E, Hara T, Okuyama T, Okumura T, Kaibori M, Kwon AH, Nishizawa M. Characterization of natural antisense transcripts expressed from interleukin lbetainducible genes in rat hepatocytes. HOAJ Biology 2012; 1: 10.

23. Kimura T, Jiang S, Nishizawa M, Yoshigai E, Hashimoto I, Nishikawa M, Okumura T, Yamada H. Stabilization of human interferon-alpha1 mRNA by its antisense RNA. Cell Mol Life Sci 2013; 70:1451-1467.

24. Dong Y, Guha S, Sun X, Cao M, Wang X, Zou S. Nutraceutical interventions for promoting healthy aging in invertebrate models. Oxid Med Cell Longev 2012; 2012: 718491.

25. Bittencourt VCB, Figueiredo RT, da Silva RB, Mourão-Sá DS, Fernandez PL, Sassaki GL, Mulloy B, Bozza MT, Barreto-Bergter E. An alpha-glucan of Pseudallescheria boydii is involved in fungal phagocytosis and Toll-like receptor activation. J Biol Chem 2006; 281: 22614-22623.

26. Geurtsen J, Chedammi S, Mesters J, Cot M, Driessen NN, Sambou T, Kakutani R, Ummels R, Maaskant J, Takata H, Baba O, Terashima T, Bovin N, VandenbrouckeGrauls CM, Nigou J, Puzo G, Lemassu A, Daffé M, Appelmelk BJ. Identification of mycobacterial alpha-glucan as a novel ligand for DC-SIGN: involvement of mycobacterial capsular polysaccharides in host immune modulation. J Immunol 2009; 183: 5221-5231.

27. Hijikawa T, Kaibori M, Uchida Y, Yamada M, Matsui K, Ozaki T, Kamiyama Y, Nishizawa M, Okumura T. Insulin-like growth factor 1 prevents liver injury through the inhibition of TNF-alpha and iNOS induction in D-galactosamine and LPS-treated rats. Shock 2008; 29: 740-747.

28. Gusarov I, Gautier L, Smolentseva O, Shamovsky I, Eremina S, Mironov A, Nudler E. Bacterial nitric oxide extends the lifespan of C. elegans. Cell 2013; 152: 818-830. 